\title{
Progression of Diabetic Retinopathy after Uncomplicated Phacoemulsification in Diabetic Patients
}

\begin{abstract}
${ }^{a}$ Department of Ophthalmology, Benha faculty of medicine, Banha University, Egypt. ${ }^{\mathrm{b}}$ Department of Ophthalmology, Shebin Elkawm Ophthalmology Hospital, Egypt.
\end{abstract}

Aida A. Hussein ${ }^{\text {a }}$, Mohammed T. Higazy ${ }^{\text {a }}$,Elham A. Gad ${ }^{\text {a }}$, Ahmed H.Khallaf ${ }^{\text {b }}$

Correspondence to: Ahmed H.Khallaf Department of Ophthalmology, Shebin Elkawm Ophthalmology Hospital, Egypt.

\section{Email:}

dr.ahmadkhallaf@gmail.com

Received: 24 January 2021

Accepted: 1 February 2021

\begin{abstract}
:
Background: Diabetes is the most common risk factor for cataract. Cataract remains one of the major causes of blindness in diabetic patients. Some studies report an increased risk of progression, whereas others report that this progression simply represents the natural course of the disease rather than a surgical outcome. Objectives: To evaluate the influence of uncomplicated unilateral phacoemulsification on postoperative progression of diabetic retinopathy in diabetic patients. Methods: Between February 2018 and August 2018, this prospective observational study included 20 diabetic patients, who were fit for unilateral phacoemulsification, of both genders aged from 35 to 80 years, with Grade I - Grade II cataract and with mild to moderate non-proliferative diabetic retinopathy. The process included 6 months of follow up with complete clinical ophthalmic examination, Fluorescein fundus angiography and Optical
\end{abstract} coherence tomography. Results: Our study included 20 patients; study report 6 males and 14 females with mean age of 58 \pm 11 years; 17 had mild NPDR and 3 had moderate NPDR. Comparing preoperative and final postoperative visit for the whole study group ,30\% showed regression of the DR (6 out of 20), 35\% had no significant change (7 out of 20) and 35\% showed progression (7 out of 20). Conclusion: Uncomplicated phacoemulsification cataract surgery may not be responsible for an accelerated rate of DR progression postoperatively. Instead, any DR progression that does occur postoperatively followed a benign course and may simply represent the natural history of the disease.

Key Words: Cataract, Diabetic Retinopathy, Phacoemulsification 


\section{Introduction:}

Diabetes Mellitus is the most common risk factor for cataract, with a three- to four-fold excess prevalence in diabetic patients older than 65 years ${ }^{(1)}$. Cataract remains one of the major causes of blindness in diabetic patients ${ }^{(2)}$.

Many researchers reported good visual improvement after cataract surgery in diabetic patients ${ }^{(3)}$. Currently, early surgery is favored before the development of significant diabetic retinopathy rather than to wait for the cataract to become denser ${ }^{(4)}$. Diabetic retinopathy has features of both microvascular occlusion and leakage ${ }^{(5)}$.

The progression of diabetic retinopathy and maculopathy after phacoemulsification surgery has been a subject of debate. Some studies report an increased risk of progression ${ }^{(6)}$, whereas others report that this progression simply represents the natural course of the disease rather than a surgical outcome ${ }^{(7)}$. Others confirmed that cataract surgery by phacoemulsification does not represent an apparent risk factor for progression of diabetic retinopathy ${ }^{(8)}$.

Multiple factors have been identified to influence diabetic retinopathy progression, including type, degree of control, course, and control pattern of diabetes, as well as hypertension and hyperlipidemia ${ }^{(9)}$.

Although the phacoemulsification had smaller harassment to the ocular structure compared with the intra-capsular or extracapsular cataract extraction, it still induced different degrees of inflammation and damaged the blood-ocular barrier with the natural defense "lens diaphragm", leading to diffusion and redistribution of the intraocular cytokines, accelerating the retinal microvascular disease, and thereby, accelerating the diabetic retinopathy progression ${ }^{(10)}$.

Furthermore, phacoemulsification has been shown to affect blood aqueous barrier significantly more severely in patients with diabetes with proliferative diabetic retinopathy, than non-proliferative diabetic retinopathy and non-diabetic patients ${ }^{(11)}$.

Patients \& Methods

- Study Design:

Prospective, non-randomized and casecontrolled study indicating any progression of diabetic retinopathy after uncomplicated phacoemulsification in diabetic patients.

- Study Site:

Most of the cases (16 out of 20) were operated in Ophthalmology department in 
Benha Faculty of Medicine and few (4 out of 20) were operated in Shebin Elkoum Ophthalmology Hospital from February 2018 to August 2018.

\section{Study Population:}

- Twenty diabetic patients, fit for unilateral phacoemulsification, were included in this study after an informed and written consent for participation.

- The approval of the hospitals' ethical committees was obtained.

- Detailed Medical and ocular history including age, gender, systemic diseases esp. Hypertension and Diabetes Mellitus, visual complaints and previous ocular surgeries.

- Complete ophthalmic examination in every visit including Visual Acuity by Snellen chart, Slit lamp biomicroscopy, IOP measurement, retinal examination, Fluorescein fundus angiography and Optical coherence tomography

\section{Inclusion Criteria:}

- Both genders aged from 35 - 80 years.
- Diabetic patients with Grade I - Grade II cataract and mild to moderate nonproliferative diabetic retinopathy.

\section{Exclusion Criteria:}

-Traumatic cataract.

- Severe Non-Proliferative/ Proliferative

Diabetic Retinopathy

- Marked Asymmetry of retinopathy

between both eyes

- Mature senile cataract or severe lens opacity

- Previous cataract surgery in the fellow eye within 12 months

- Potentiality to have Phacotrabeculectomy surgery

- Complications during or after the surge

\section{Results}

- The mean Age of the studied group was $58 \pm 11$ years (ranged from 39 to 80 ) (Table 1)

- Regarding the Gender: 16 were females and 6 were males

- Regarding the Diagnosis: 17 had mild NPDR and 3 had moderate NPDR; $85 \%$ to $15 \%$ respectively (Figure 1 )

Table (1) shows age distribution of the studied group:

Age distribution

Age (years)
Mean \pm SD

Range
$58 \pm 11$

$39-80$ 


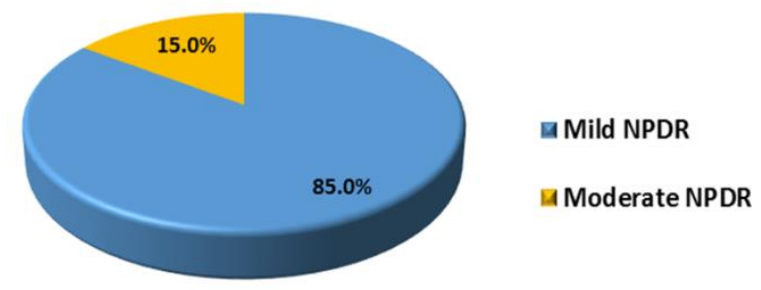

Figure (1): Mild to Moderate NPDR ratio

Regarding the Best Corrected Visual Acuity (BCVA) throughout the study:

-The mean BCVA of the studied group in February was $0.36 \pm 0.09(0.2-0.5)$

- The mean BCVA of the studied group in March was $0.35 \pm 0.1(0.2-0.5)$

- The mean BCVA of the studied group in May was $0.36 \pm 0.09(0.2-0.5)$
- The mean BCVA of the studied group in August was $0.47 \pm 0.15(0.1-0.66)$

- Comparing preoperative and final postoperative BCVA, there's significant difference: $0.36 \pm 0.09$ and $0.47 \pm 0.15$ respectively ( $\mathrm{p}$ value $<0.05$ ) and the mean difference is 0.9 (Figure 2)

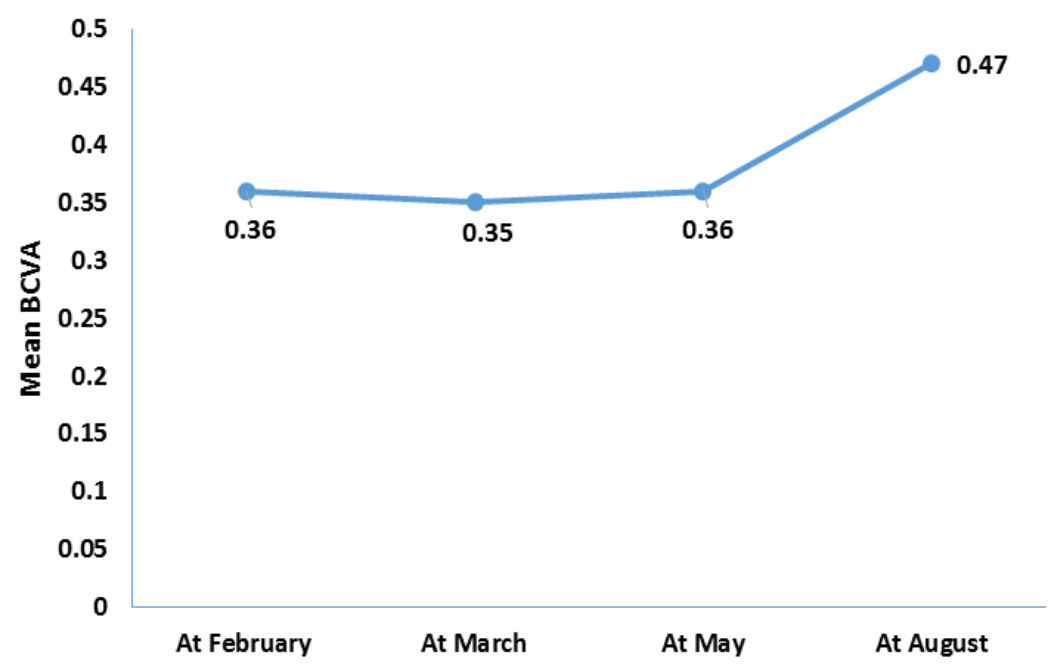

Figure (2): Mean BCVA preoperative and in follow-up visits 
Regarding the Intra ocular pressure (IOP) throughout the study:

- The mean IOP of the studied group in February was $20 \pm 3 \mathrm{mmHg}(14-25)$

- The mean IOP of the studied group in March was $17 \pm 2 \mathrm{mmHg}(12-21)$

- The mean IOP of the studied group in May was $19 \pm 3 \mathrm{mmHg}(13-25)$

- The mean IOP of the studied group in August was $20 \pm 4 \mathrm{mmHg}(14-29)$

- Comparing preoperative and final postoperative IOP, there's no significant difference: $20 \pm 3$ and $20 \pm 4$ respectively (Figure 3)

- Regarding the Central macular thickness (CMT) throughout the study:

- The mean CMT of the studied group in February was $265.6 \pm 24.6$ (226.1-320.3)

- The mean CMT of the studied group in March was $279.1 \pm 26.8$ (229.6 - 328.6)

- The mean CMT of the studied group in May was $283.3 \pm 50.8(230-468.2)$

- The mean CMT of the studied group in August was $274.7 \pm 45.8(180.3-430.4)$
- Comparing preoperative and final postoperative CMT, there's no significant difference: $265.6 \pm 24.6$ and $274.7 \quad \pm 45.8$ respectively

- Regarding the Frequency distribution of Fluorescein leakage throughout the study:

- In March; $1^{\text {st }}$ FU after the OP: 20\% (4 out of 20) of the patients developed CNVs

- In May; $2^{\text {nd }} F U$ : The Fundus of those 4 patients showed regression and disappearance of such CNVs

- In August; final FU: Fundus of 3 more patients showed improvement with no leakage

- Comparing preoperative and final postoperative visit, there's no significant difference

Comparing preoperative and final postoperative visit for the whole study group, 30\% showed regression of the DR (6 out of 20), 35\% had no significant change (7 out of 20) and 35\% showed progression (7 out of 20) (Figure 4)

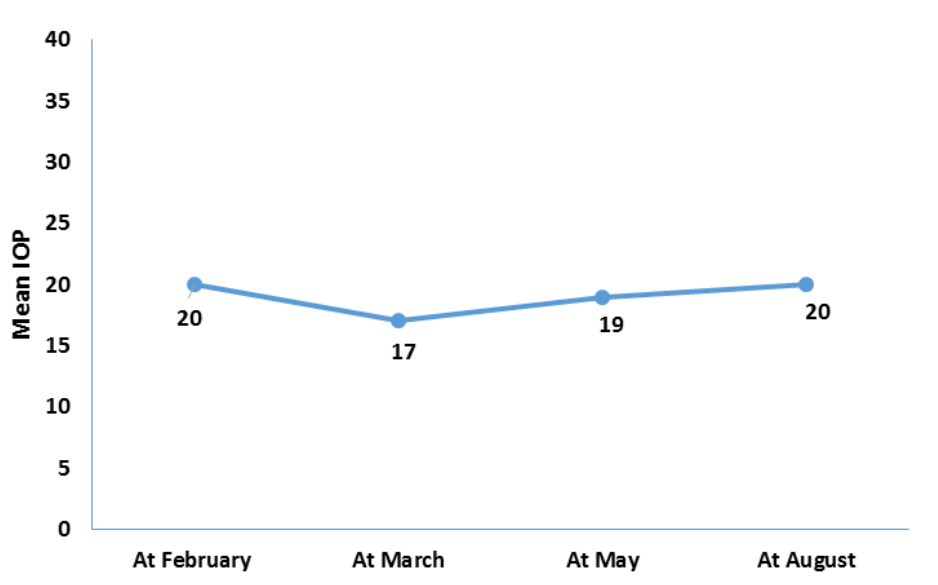

Figure (3): Mean IOP preoperative and in follow-up visits 


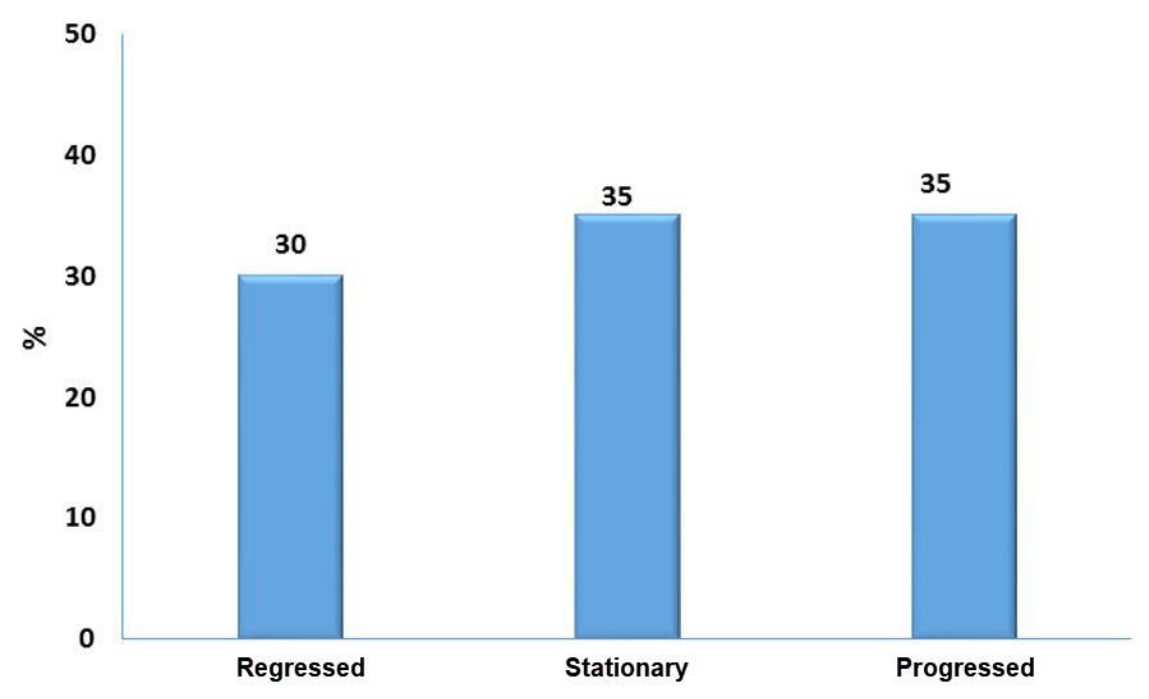

Figure (4): Progression of DR for the whole study group

\section{Discussion}

Cataract in diabetic patients not only decreases the vision but also prevents adequate fundus examinations and optimal therapy. Therefore, cataract surgery has meanings of visual rehabilitation, diagnostic and therapeutic purposes to diabetic patients (12). The present study was conducted to detect any progression of diabetic retinopathy following uncomplicated phacoemulsification in cataractous diabetic patients.

One researcher reported that diabetic patients without retinopathy were more likely to show progression of retinopathy than patients with mild-to-moderate DR ${ }^{(8)}$ but others reported that NPDR was the strongest factor associated with retinopathy progression ${ }^{(13)}$.

In this study assessment of macular edema was carried out using fluorescein angiography and optical coherence tomography preoperative and in each follow up visit up to 6 months postoperatively; worsening of the condition occurred in only 7 eyes (35\%).

After six months postoperative, the final CMT increased by $3 \%$ more than the preoperative CMT which was statistically insignificant difference ( $\mathrm{p}$-value $=0.365$ ).

We concluded that the degree of diabetic macular edema temporarily worsened after cataract surgery. 
Our results agree with previous studies which showed that eyes with pre-operative DME prior to cataract surgery are at higher risk for developing central-involved $\mathrm{ME}^{(14)}$. In one study the risk of ME was associated with preoperative grade of retinopathy; the risk of DME in the 1st year postoperatively was $1.0 \%$ (no DR preoperatively), $5.4 \%$ (mild non-PDR [NPDR]), 10.0\% (moderate NPDR), $13.1 \%$ (severe NPDR), and $4.9 \%$ (PDR). This large real-world study proves that the risk of treatment requiring DME increases sharply in the 1 st year after cataract surgery and that those with moderate and severe NPDR are most at risk of such progression ${ }^{(15)}$.

A prospective study was carried out to quantitatively examine changes in macular edema after phacoemulsification surgery in eyes with and without diabetic retinopathy. 34 eyes with DR and 34 eyes with no DR scheduled for phacoemulsification surgery were recruited. Foveal thickness and macular volume were measured using optical coherence tomography preoperatively and at 3,6 , and 12 months postoperatively. Grade of diabetic macular edema was also examined. Preoperatively, there were no significant differences between groups in foveal thickness and macular volume ${ }^{(16)}$.
The foveal thickness increased by $20.3 \%$ in the DR group and by $6.0 \%$ in the non-DR group at 3 months after surgery, but thereafter decreased gradually. The grade of diabetic macular edema also progressed in $24 \%$ of the eyes with DR. However, (33.3\%) of macular edema that occurred after surgery in eyes with DR had resolved spontaneously by 12 months. These results suggest that the change in macular edema in some diabetic patients is transient, while in other diabetics, the macular edema is substantial, with marked progression of maculopathy ${ }^{(16)}$.

In accordance with the present study, they postulated that the degree of diabetic macular edema generally worsens after cataract surgery. However, macular edema that occurs after cataract surgery resolves spontaneously in some patients for up to a year.

In another study, it was stated that the changes in CMT values may be due to the inflammatory response to uncomplicated phacoemulsification at postoperative 1 month and may also be associated with the pathologic features of Irvine-Gass syndrome caused by cytokines and growth factor (e.g., prostaglandins and VEGF) released from the blood-ocular barrier after cataract surgery. Surgery itself can cause inflammatory response by releasing prostaglandins, which plays an important role in the occurrence of 
macular thickening. The results indicate that uncomplicated phacoemulsification has some effect upon the underlying pathophysiology of retinopathy ${ }^{(17)}$.

In our study we found that 5 cases $(25 \%)$ showed progression of mild DR to moderate DR, 2 cases (10\%) showed progression of moderate DR to severe NPDR.

Another study demonstrated that $18 \%$ of diabetic patients developed increases in CSMT > 30\% after cataract surgery. Its peak incidence was at 1-month post-surgery and it resolved spontaneously in $68 \%$ of patients by 6 months post-surgery. However, macular edema did not affect the severity of diabetic retinopathy ${ }^{(18)}$.

Similarly, it was concluded that cataract surgery significantly increases the risk of NPDR development. Its impact persists 5 years after surgery. The risk developing NPDR post-operatively are even higher if there are complications during cataract surgeries. Diabetic patients receiving cataract surgery who have comorbidities are more susceptible to NPDR development ${ }^{(8)}$. Contrarily, in another review it was reported that recent studies do not support the generalized conclusion that phacoemulsification causes progression of retinopathy and $\mathrm{ME}$ in all diabetic patients (19).

Our rates of progression, 6 months' study, were lower than that reported by another study which studied progression of diabetic retinopathy (DR) after phacoemulsification in diabetic patients for 12 months. An increase was reported in the doubling rate of DR progression 12 months after surgery ${ }^{(20)}$.

Notably, a meta-analysis of different pairedeye studies showed that the progression rate of DR and the incidence rate of DME increased significantly after phacoemulsification ${ }^{(21)}$.

In accordance with a control-based study, it was found that progression of DR occurred at about $23.9 \%$ (11/46) in the operated eyes and $19.6 \%(9 / 46)$ in the non-operated eyes. Therefore, cataract surgery by phacoemulsification does not represent an apparent risk factor for progression of DR (22).

In our study, $70 \%$ of cases showed improvement by 1 or more lines in their visual acuity, while $15 \%$ of cases showed no change and $15 \%$ showed worsening in their visual acuity. The mean postoperative best corrected visual acuity at final follow up visit was $(0.47 \pm 0.15)$ 
In another study, postoperative visual acuity increased by 2 or more lines in 50 patients $(83 \%)$; it was better than 0.5 in $80 \%{ }^{(23)}$. In accordance with another study, statistically significant improvements were observed in postoperative BCVA after three months after phacoemulsification ${ }^{(24)}$.

Therefore, we concluded that it is not good to defer cataract surgery in diabetic patients with no DR or with only mild to moderate NPDR.

In another study it was concluded that both visual acuity and the vision related quality of life of the patients with diabetic retinopathy improved significantly after cataract surgery. Cataract surgery is an effective intervention for patients with stabilized diabetic retinopathy ${ }^{(24)}$.

Other studies concluded that uncomplicated phacoemulsification cataract surgery seems to exacerbate the progression of DR in north African patients with worse controlled diabetes. Poorer visual outcome is observed in patients developing macular edema ${ }^{(23)}$. Currently, early surgery is favored before the development of significant DR rather than to wait for the cataract to become denser. All efforts should be made to stabilize DR using laser treatment before cataract surgery. Larger prospective studies are needed to better confirm the relationship between phacoemulsification and postoperative progression of DR.

\section{Conclusion}

Our findings suggest that uncomplicated phacoemulsification cataract surgery may not be responsible for an accelerated rate of DR progression postoperatively. Instead, our findings suggest that any DR progression that does occur postoperatively may simply represent the natural history of the disease. We found that although macular edema was common after cataract surgery, it often followed a benign course and any progression that is observed postoperatively probably represents natural progression rather than being a direct effect of surgery. Therefore, we think it is not good to defer cataract surgery in diabetic patients with no DR or with only mild to moderate NPDR. Currently, early surgery is favored before the development of significant DR rather than wait for the cataract to become denser. All efforts should be made to stabilize DR using laser treatment and/or intravitreal injection with anti VEGF before cataract surgery.

\section{References:}

1 Pollreisz A. and Schmidt-Erfurth U. Diabetic Cataract-Pathogenesis, Epidemiology and 
Treatment. Journal of Ophthalmology. 2010; 13:608-751.

2 Javadi MA. and Zarei-Ghanavati S. Cataracts in Diabetic Patients: A Review Article. Journal of Ophthalmic \& Vision Research. 2008; 3(1):52-65.

3 Krepler K., Biowski R., Schrey S., Jandrasits K. and Wedrich A. Cataract surgery in patients with diabetic retinopathy: visual outcome, progression of diabetic retinopathy, and incidence of diabetic macular edema. Graefes Arch Clin Exp Ophthalmol. 2002; 240:735-738

4 Ali KG., Soliman TT. and Mohammed AA. Effect of cataract surgery on diabetic retinopathy. Benha Med J 2015; 32:92-5

5 El-Sobky, H., El-Sebaey A., El-Hagaa A. and Gaber NK. "Evaluation of the progression of diabetic retinopathy after phacoemulsification." Menoufia Medical Journal. 2014; 27(4): 643-649.

6 Rashid S. and Young L. H. "Progression of Diabetic Retinopathy and Maculopathy After Phacoemulsification Surgery." International Ophthalmology Clinics. 2010; 50(1):155-166.

7 Flesner P., Sander B., Henning V., Parving HH, Dornonville de la Cour $\mathrm{M}$ and Lund-Andersen $\mathrm{H}$. Cataract surgery on diabetic patients. A prospective evaluation of risk factors and complications. Acta Ophthalmol Scand. 2002; 80:19-24

8 Jeng CJ., Hsieh YT., Yang CM., Yang CH, Lin CL and Wang IJ. Development of diabetic retinopathy after cataract surgery. PLoS ONE. 2018; 13(8): e0202347.

9 Sayin N., Kara N. and Pekel G. Ocular complications of diabetes mellitus. World Journal of Diabetes. 2015; 6(1):92-108.

10 Tu Y., Xu L., Wei W.B., Wang S, Wang YX and Jonas JB. Progression of diabetic retinopathy: the Beijing Eye Study. Chin Med J .(Engl) 2011; $124: 3635-3640$
11 Liu Y., Luo L., He M. and Liu X. Disorders of the blood-aqueous barrier after phacoemulsification in diabetic patients. Eye. 2004; 18:900-904

12 Vieira-Potter VJ, Karamichos D and Lee DJ. Ocular Complications of Diabetes and Therapeutic Approaches. Biomed Res Int. 2016; 2016:3801570.

13 Harris Nwanyanwu K, Talwar N, Gardner TW, Wrobel JS, Herman WH and Stein JD. Predicting development of proliferative diabetic retinopathy. Diabetes Care. 2013; 36(6):1562-8.

14 Chen XY, Song WJ and Cai HY. Macular edema after cataract surgery in diabetic eyes evaluated by optical coherence tomography. Int J Ophthalmol. 2016; 9(1):81-85.

15. Denniston AK, Chakravarthy U, Zhu H, Lee AY, Crabb DP, Tufail A, et. al. The UK Diabetic Retinopathy Electronic Medical Record (UK DR EMR) Users Group, Report 2: real-world data for the impact of cataract surgery on diabetic macular oedema. Br J Ophthalmol. 2017 Dec; 101(12):16731678.

16 Hayashi K, Igarashi C, Hirata A and Hayashi H. Changes in diabetic macular oedema after phacoemulsification surgery .Eye (Lond). 2009 Feb; 23(2):389-96.

17 Liu J, Jones RE, Zhao J, Zhang J and Zhang F. Influence of Uncomplicated Phacoemulsification on Central Macular Thickness in Diabetic Patients: A Meta-Analysis. PLoS ONE 2015; 10(5): e0126343.

18 Kwon SI, Hwang DJ, Seo JY and Park IW. Evaluation of changes of macular thickness in diabetic retinopathy after cataract surgery. Korean J Ophthalmol. 2011;25(4):238-242.

19 Shah AS and Chen SH. Review Cataract surgery and diabetes .Curr Opin Ophthalmol. 2010 Jan; 21(1):4-9.

20 Hong T, Mitchell P, de Loryn T, Rochtchina E, Cugati $\mathrm{S}$ and Wang JJ. Development and 
Benha medical journal vol. 39, special issue (ophthalmology), 2022

progression of diabetic retinopathy 12 months after phacoemulsification cataract surgery . Ophthalmology. 2009 Aug; 116(8):1510-4

21 Wang SX, Xu Q, Du Y. H. and Wu X. Y. Does phacoemulsification speed the progression of diabetic retinopathy? A meta-analysis. International Journal of Clinical and Experimental Medicine. 2016; 9(6):8874-82.

22 Chéour M, Mazlout H and Falfoul Y. Évolution de la rétinopathie diabétique après chirurgie de la cataracte par phacoémulsification [Progression of diabetic retinopathy after cataract surgery by phacoemulsification]. J Fr Ophtalmol. 2013; 36(1):62-65

23 Maalej A., Khallouli A., Daldoul N., Wathek C. and Rannen R. "Diabetic Retinopathy Progression and Visual Outcome after Phacoemulsification: A Prospective Study". ECOphthalmology 2018; 9.5: 312-319.

24 Zhu B, Ma Y, Lin S and Zou H. Vision-related quality of life and visual outcomes from cataract surgery in patients with vision-threatening diabetic retinopathy: a prospective observational study. Health Qual Life Outcomes. 2017; 15(1):175.

To cite this article: Aida A. Hussein, Mohammed T. Higazy, Elham A. Gad, Ahmed H.Khallaf. Progression of Diabetic Retinopathy after Uncomplicated Phacoemulsification in Diabetic Patients. BMFJ 2022;39 (ophthalmology):1-11. DOI: 10.21608/bmfj.2021.59550.1374 